\title{
Calreticulin is a novel independent prognostic factor for oral squamous cell carcinoma
}

\author{
KOJI HARADA ${ }^{1}$, TAKANORI TAKENAWA ${ }^{1}$, TARANNUM FERDOUS ${ }^{1}$, \\ YASUHIRO KURAMITSU $^{2}$ and YOSHIYA UEYAMA ${ }^{1}$
}

\author{
Departments of ${ }^{1}$ Oral and Maxillofacial Surgery and ${ }^{2}$ Biochemistry and Functional Proteomics, \\ Yamaguchi University Graduate School of Medicine, Ube, Yamaguchi 755-8505, Japan
}

Received September 11, 2016; Accepted March 7, 2017

DOI: $10.3892 / \mathrm{ol} .2017 .6062$

\begin{abstract}
We focused on the expression of Calreticulin (CALR) in oral squamous cell carcinoma (OSCC) on the basis of proteomic differential display analysis data. We used QR-32 cells in this study which is a regressive murine fibrosarcoma cell clone; and QRsP-11, a progressive malignant tumor cell clone originated from QR-32. CALR is an endoplasmic reticulum luminal $\mathrm{Ca}^{2+}$-binding chaperone protein, which is thought to affect the tumor behavior of various malignancies. This study was aimed to determine the usefulness of CALR as a prognostic factor in patients with OSCC. We investigated the expression of CALR in tissue samples taken from 111 OSCC patients by immunohistochemistry, and we also analyzed the relationship between CALR expression and patients' clinicopathological characteristics as well as patient survival. Positive immunohistchemical staining of CALR was observed in the cancer cell cytoplasm. Among 111 patients, high expression of CALR was observed in 44 patients $(39.6 \%)$, whereas low expression was observed in 67 patients $(60.4 \%)$. Significant association was found between CALR expression and $\mathrm{T}$ classification $(\mathrm{P}=0.0027), \mathrm{N}$ classification $(\mathrm{P}=0.0219)$, stage $(\mathrm{P}=0.0013)$, and patient outcome $(\mathrm{P}=0.0014)$. Log-rank test showed that, there is a significant difference $(\mathrm{P}<0.0001)$ in 5-year survival rates between patients showing CALR high-expression (59.1\%) and CALR low-expression (86.6\%). According to our Multivariate analysis, reduced term survival of patients was correlated to high levels of CALR expression $(\mathrm{P}<0.0001)$. Our findings suggest that elevated expression of CALR might play an important role in tumor progression in OSCC, and could be considered as a useful prognostic factor in OSCC patients.
\end{abstract}

Correspondence to: Dr Koji Harada, Department of Oral and Maxillofacial Surgery, Yamaguchi University Graduate School of Medicine, 1-1-1 Minamikogushi, Ube, Yamaguchi 755-8505, Japan E-mail: harako@yamaguchi-u.ac.jp

Key words: calreticulin, prognostic marker, oral squamous cell carcinoma

\section{Introduction}

Oral squamous cell carcinoma (OSCC) caused 364,872 deaths (216,975 men and 147,897 women) in Japan in 2013 (1). Approximately $60 \%$ of head and neck cancer patients are in the advanced stage (stage III and stage IV) of the disease $(2,3)$. As OSCCs are often diagnosed at the advanced stage, survival rate of the patients is low. Also, currently available clinical diagnosis, evaluation and advanced treatment methods could not ensure increased survival rate of the patients $(2,3)$. Therefore, we should continue to investigate for suitable biomarkers for early diagnosis or prognostic prediction of OSCC. The development of useful biomarkers must lead to the discovery of the novel therapeutic strategy or new chemo-preventive agents.

The most crucial features of malignant tumors may be non-predictable progression. Briefly, rapid growth and proliferation rate, high invasive capacity and metastatic ability are the characteristics of progressive tumor cells, while regressive benign tumor cells show opposite characteristics. Progressive (QRsP-11 clone) and regressive (QR-32 clone) murine fibrosarcoma tumor models were successfully established by Okada et al $(4,5)$. The progressive clone QRsP-11 with malignant characteristic was established from QR-32 regressive clone, which is a weakly tumorigenic and non-metastatic cell clone. In normal syngeneic mice, QR-32 cells were reported to regress spontaneously if the cells were injected intravenously $\left(1 \times 10^{6}\right.$ cells) or subcutaneously (up to $2 \times 10^{5}$ cells). However, subcutaneous injection of these cells with co-implanted gelatin sponge results in progressive growth of the cells. From these progressively-growing cells, QRsPs cell lines were established which has the ability to progressively grow in mice without the presence of gelatin sponge. QRsPs, e.g., -QRsP-11 is as malignant tumor cell clone which is more tumorigenic and metastatic. Proteomic studies of QR-32 and QRsP-11 cells were conducted by two-dimensional gel electrophoresis (2-DE) to evaluate the differential expression patterns of proteins between these cell types in order to understand various important factors in tumor progression (6,7). Differential display analysis of the nuclear protein expression of these cells revealed eight differentially-regulated nuclear proteins between QR-32 and QRsP-11, and Zing finger protein ZXDC is one of them (6). In addition, 11 differentially regulated proteins were found 


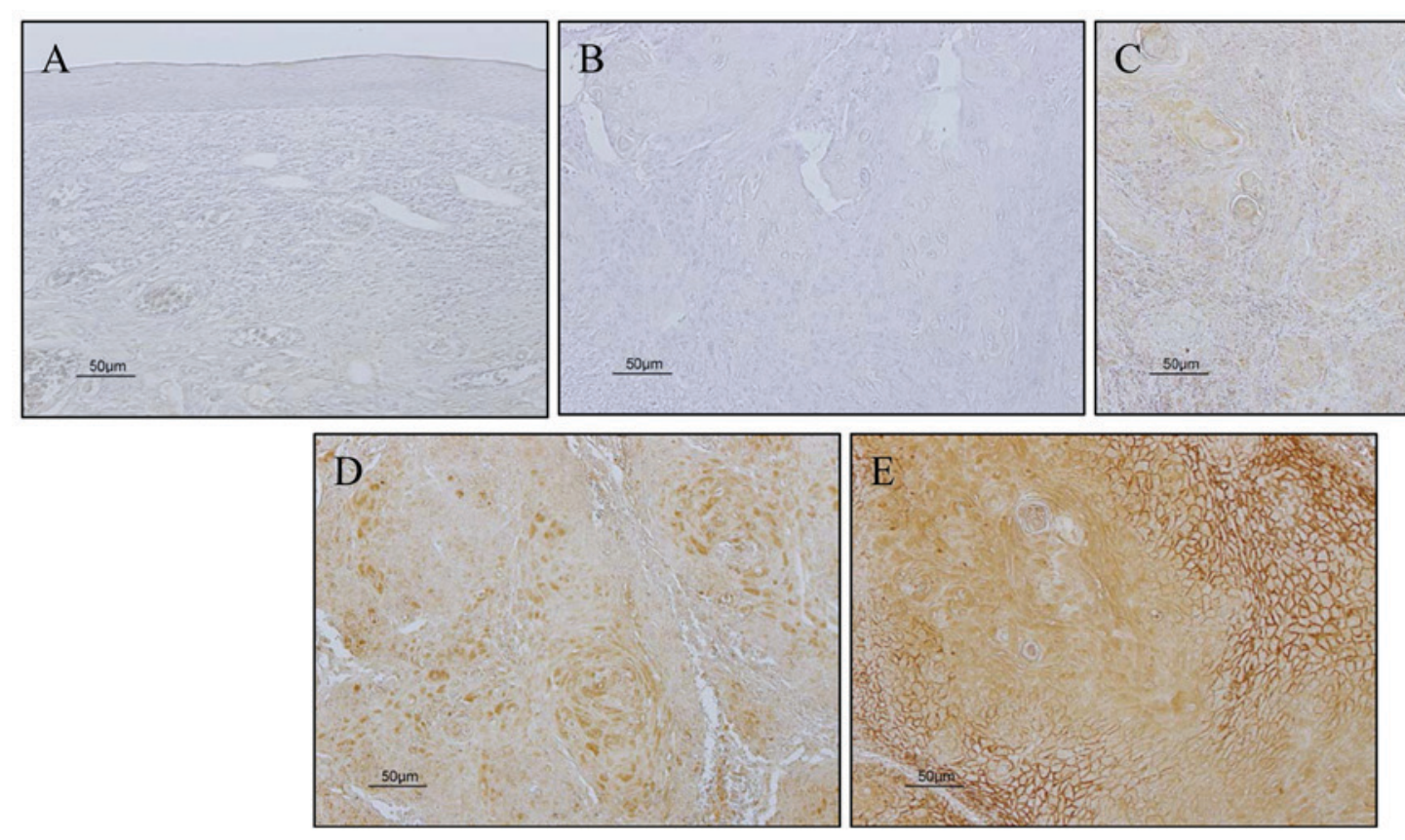

Figure 1. CALR expression in normal tissue and OSCC. (A) Immunohistochemical staining showed negative CALR expression in normal epithelial cellular cytoplasm. (B) OSCC cells with negative CALR expression. (C) Weak positive immunoreaction of CALR was detected in the cytoplasm. (D) Moderate positive immunoreaction of CALR was observed in the cytoplasm. (E) Strong positive immunoreaction was detected both in the cytoplasm and cell membrane.

by proteomic differential display analysis of cytoplasmic proteins, including heat-shock protein (HSP)-90 (8). In the present study, we focused on Calreticulin (CALR) which is one of those 11 proteins.

CALR is a $46 \mathrm{kDa}$ protein which acts as a molecular chaperone and a major $\mathrm{Ca}(2+)$-binding (storage) protein in the endoplasmic reticulum (ER) that ensures proper folding of glycoproteins (9-11). CALR controls a number of diverse biological processes in ER and other parts of the cell; such as gene expression, transcriptional activities, cell proliferation, phagocytosis, apoptosis and immune response $(9,10)$. It can also affect cell adhesion, wound healing and migration $(9,10)$. Although there are many studies on the role of CALR in a variety cellular processes and functions, its role on human carcinogenesis still remains unclear (11).

There have been studies on the alteration of CALR expression in lung cancer, ovarian cancer, gibloblastoma, gastrointentinal cancer, breast cancer, urinary tract cancer, which indicates its role in promoting or suppressing various types of cancers (9-11). Overespression of CALR has been associated with a number of malignancies together with several types of cancers (12-15). Elevated expressions of CALR in the sera of patients with autoimmune diseases were also reported (12). Again, differential expression of CALR was reported in some cancer types, and in some cases CALR low-expression was found to promote cancer growth $(11,12)$. In lung cancer, the expression level of CALR on tumor cell membrane was apparently related with the pathological grade of the tumor (12). Positive immunohistochemical staining of CALR was associated with lymph node metastasis and high microvessel density in gastric cancer; and resulted in poor patient survival (15). However, the importance of CALR expression in the regulation of OSCC tumor growth or malignancy is largely unknown. The purpose of this study was to determine whether CALR expression could be a useful prognostic factor in patients with OSCC.

\section{Materials and methods}

Patients and patient samples. This study was carried out with tissue specimens collected from 111 OSCC patients. All these patients were treated at Yamaguchi University Hospital from April 2000 to March 2010 and underwent curative surgery. Post-operative chemoradiation was used in the case of tumors with positive surgical margin, and post-operative radiation or chemoradiation was used for extranodal invasion. Surgical resection alone was carried out for 93 cases, surgical resection plus radiation for 6 cases, and surgical resection plus chemoradiation for 12 cases. Primary oral cancer tissue samples were obtained by biopsy before the patients received any treatment. Phosphate-buffered $10 \%$ formalin was used to fix the tissue samples and then the tissues were embedded in paraffin blocks.

The tumors were staged according to the Tumor, Node and Metastasis (TNM) classification of the Union Internationale Contra le Cancer (UICC) (2002). Institutional Review Board (IRB) approval from the Ethics Committee of the Yamaguchi University Hospital was obtained for this study (Ref. H28-057). This being a retrospective study, informed consent was waived by the IRB.

Immunohistochemical staining and the analysis of staining. Formalin fixed and paraffin embedded (FFPE) samples were used for the immunohistochemical analysis. FFPE tissue blocks were sliced into $4-\mu \mathrm{m}$ thick sections and attached on slides. These FFPE slides were dried, then deparaffinized in xylene and immersed in graded series (100-70\%) of ethanol concentrations. After phosphate buffered saline wash, the sections were heated in a microwave oven in an antigen 
retrieval solution. The sections were allowed to cool down and immersed in $0.3 \%$ hydrogen peroxide solution in methanol for $30 \mathrm{~min}$ to quench any residual peroxidase activity. After incubation with Dako REAL ${ }^{\mathrm{TM}}$ peroxidase-blocking solution (S2023; Dako, Glostrup, Denmark) for $15 \mathrm{~min}$, the sections were incubated overnight at $4^{\circ} \mathrm{C}$ with rabbit polyclonal anti-Calreticulin antibody (StressMarq Bioscience Inc., Victoria, BC, Canada). Then the sections were incubated with a secondary antibody solution for $60 \mathrm{~min}$ at room temperature, followed by diaminobenzidine using REAL ${ }^{\mathrm{TM}}$ Envision $^{\mathrm{TM}}$ Detection system kit (K5007, Dako). The sections were lightly counterstained with hematoxylin, dehydrated in graded series of ethanol, immersed in xylene and coverslipped.

We evaluated CALR expression in our samples according to the method described by Kuramitsu et al with some modification (6). Briefly, we compared the intensity of CALR staining in cancer cells from cancerous tissue with that in the normal oral epithelium. The percentage of positive cells was graded as $0,0 \%$ immunopositive cells; $1,<50 \%$ positive cells; $2, \geq 50 \%$ positive cells. And the staining intensity was graded as 0 , negative; 1 weak; 2 , moderate; 3 , strong (Fig. 1 ). The sum of the assigned values of the percentage of positive cells and the staining intensity was regarded as the immunoreactivity score. Scores between 0-2 were regarded as low expression, scores between 3-5 were regarded as high expression. Immunoreactivity for CALR expression was evaluated by three authors (K.H., T.T. and T.F.), who had no knowledge of the patient's clinical status.

Statistical analysis. We used Chi-square test to determine the association of CALR expression in the OSCC tissues with the clinical and pathological variables. Overall survival (OS) and event-free survival (EFS) were defined as the time from treatment initiation to the date of death from any cause. To estimate the probability of OS or EFS as a function of time, Kaplan-Meier method was used. Log-rank test was used to compare the statistical differences in the survival of subgroups of patients. To study the effects of CALR expression on the OS, a multivariate survival analysis was carried out using the Cox regression model. All P-values were based on two-tailed statistical analysis, and P-values $<0.05$ were considered statistically significant $(\mathrm{P}<0.05$ and $\mathrm{P}<0.01)$. All statistical analysis was done using the StatView software (version 5.0 J; SAS Institute Inc., Cary, NC, USA).

\section{Results}

Patients and tumor characteristics. Table I shows the characteristics of patients with OSCCs used in this study. This study included a total of 111 OSCC patients. The median age of the patients was 70 years (range, 18-96 years). The median follow-up time was 5.4 years. We diagnosed 26 patients with stage I, 41 with stage II, whereas stage III and IV were diagnosed in 12 and 32 patients, respectively.

CALR expression in tumor cells and clinicopathological features. Table II shows the correlation between CALR expression in tumor cell and clinicopathological factors of patients. Among 111 patients with OSCC, we observed low expression of CALR in the tumor cells of 67 patients $(60.4 \%)$
Table I. Patient characteristics.

\begin{tabular}{|c|c|c|}
\hline Characteristics & $\begin{array}{l}\text { Total }(\mathrm{n}=111) \\
\text { No. of patients }\end{array}$ & $\%$ \\
\hline \multicolumn{3}{|l|}{ Age (years) } \\
\hline Median & 70.0 & \\
\hline Min-mac & $18-96$ & \\
\hline$<65$ & 41 & 36.9 \\
\hline$>65$ & 70 & 63.1 \\
\hline \multicolumn{3}{|l|}{ Gender } \\
\hline Male & 60 & 54.1 \\
\hline Female & 51 & 45.9 \\
\hline \multicolumn{3}{|l|}{$\mathrm{T}$ classification } \\
\hline 1 & 26 & 23.4 \\
\hline 2 & 55 & 49.5 \\
\hline 3 & 8 & 7.2 \\
\hline 4 & 22 & 19.8 \\
\hline \multicolumn{3}{|l|}{$\mathrm{N}$ classification } \\
\hline 0 & 82 & 73.9 \\
\hline 1 & 12 & 10.8 \\
\hline 2 & 14 & 12.6 \\
\hline 3 & 3 & 2.7 \\
\hline \multicolumn{3}{|l|}{ Stage } \\
\hline I & 26 & 23.4 \\
\hline II & 41 & 36.9 \\
\hline III & 12 & 10.8 \\
\hline IV & 32 & 28.8 \\
\hline \multicolumn{3}{|l|}{ Outcome } \\
\hline Alive & 84 & 75.7 \\
\hline Death & 27 & 24.3 \\
\hline \multicolumn{3}{|c|}{$\begin{array}{l}\text { CALR expression in } \\
\text { tumor cells cytoplasm }\end{array}$} \\
\hline Low & 67 & 60.4 \\
\hline High & 44 & 39.6 \\
\hline
\end{tabular}

and high in 44 patients (39.6\%). Positive immunoreactivity of CALR in tumor cells was significantly associated with $\mathrm{T}$ classification $(\mathrm{P}=0.0027), \mathrm{N}$ classification $(\mathrm{P}=0.0219)$, higher stage $(\mathrm{P}=0.0013)$ and fatal outcome $(\mathrm{P}=0.0014)$. However, gender and age of the patients were not correlated with CALR positivity.

CALR expression in OSCCs and patient survival. The overall median follow-up of the cohort was 5.4 years, 27 patients died. In addition, 11 patients had events including recurrences and/or post-operative metastasis. Five-year OS and EFS for the whole population patients were 75.7 and $65.8 \%$, respectively. The 5-year OS rates of CALR high- and low-expression tumors were 59.1 and $86.6 \%$, respectively. Also, the 5-year EFS rates of CALR high- and low-expression tumors were 43.21 and $80.6 \%$, respectively. Log-rank test showed significant difference $(\mathrm{P}<0.0001)$ in the OS rate between patients with high and low CALR expression. In addition, CALR expression 
Table II. Correlation of CALR expression and clinicopathological factors in OSCC.

CALR expression in tumor cell

\begin{tabular}{cccc} 
Characteristics & $\begin{array}{c}\text { Low expression } \\
(\mathrm{n}=67,60.4 \%)\end{array}$ & $\begin{array}{c}\text { High expression } \\
(\mathrm{n}=44,39.6 \%)\end{array}$ & $\begin{array}{c}\text { Total } \\
(\mathrm{n}=111)\end{array}$ \\
\hline
\end{tabular}

Age (years)

$<65 \quad 25$

$>65$

25

Gender

Male

Female 39

28

T classification

$1+2$

$3+4$

56

11

$\mathrm{N}$ classification

0

$1+2+3$

54

13

Stage

I+II

III+IV

Outcome

Alive

Death
58

9
16

28

21

23

25

19

28

16

19

25

26

18
0.7247

41

70

60

51

0.0027

81

30

82

29

0.0013

67

44

0.0014

84

27

${ }^{a} \mathrm{P}<0.05$ was defined as significant.

CARL tumor positivity

(\% positive neoplastic cell)
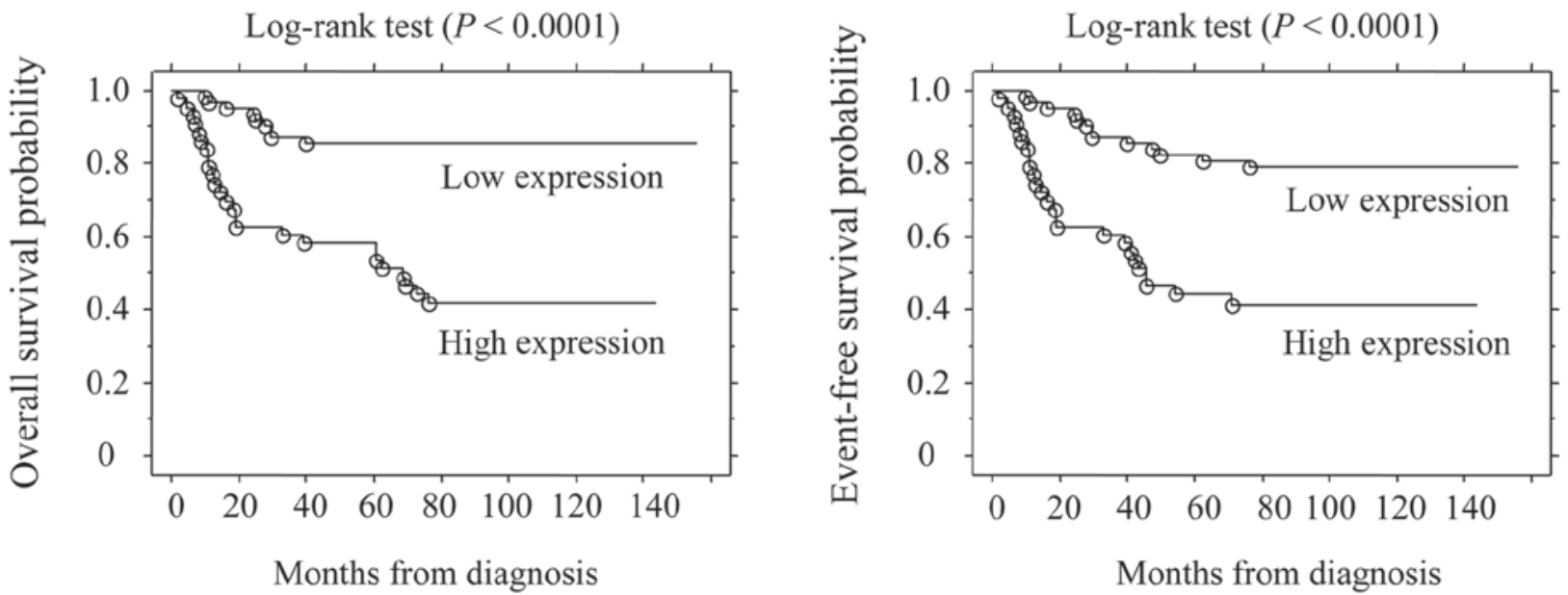

Figure 2. Kaplan-Meier curves for overall survival (OS) and event-free survival (EFS) according to CALR expression in patients with OSCC. Patients with CALR high expression had a lower OS than patients with CALR low expression (log-rank test, P<0.0001). Similarly, patients showing CALR high expression had lower EFS than patients with CALR low expression (log-rank test, $\mathrm{P}<0.0001)$.

also was associated with EFS ( $\mathrm{P}<0.0001)$ (Fig. 2). Moreover, multivariate analysis also revealed that $\mathrm{T} 3+\mathrm{T} 4(\mathrm{P}=0.0226)$, Stage $(\mathrm{P}=0.0048), \mathrm{N}$ positive $(\mathrm{P}=0.0013)$ and high expression of CALR $(\mathrm{P}<0.0001)$ was a predictor of reduced survival, although no other varieties were identified (Table III).

\section{Discussion}

Calreticulin (CALR), an endoplasmic reticulum (ER)-resident protein, plays an important role in multiple biological processes such as $\mathrm{Ca}^{2+}$ homeostasis (9). CALR regulates cell 
Table III. Risk factors affecting overall survival rate determined by Cox's proportional hazard model.

\begin{tabular}{lccc}
\hline Variables & Hazard ratio & $95 \%$ CI & P-value $^{\mathrm{a}}$ \\
\hline $\begin{array}{l}\text { Age (years) } \\
<65 \text { vs. }>65\end{array}$ & 1.652 & $0.771-3.542$ & 0.1966 \\
$\begin{array}{l}\text { Gender } \\
\text { Male vs. female }\end{array}$ & 1.351 & $0.689-2.650$ & 0.3814 \\
$\begin{array}{l}\text { T classification } \\
\text { T1+T2 vs. T3+T4 }\end{array}$ & 2.216 & $1.118-4.391$ & 0.0226 \\
N classification & & & 0.0013 \\
$\quad$ N0 vs. N1+N2+N3 & 3.041 & $1.543-5.995$ & 0.0048 \\
Stage & & & $<0.0001$ \\
I+II vs. III+IV & 2.673 & $1.349-5.296$ & \\
CALR expression & & $2.624-12.130$ & \\
High vs. low & 5.641 & &
\end{tabular}

CI, confidence interval. ${ }^{\mathrm{P}}<0.05$ was defined as significant.

proliferation, wound healing, immune response, apoptosis and may other important cellular processes $(9,10)$. However, the role of CALR in OSCC development has not been fully clarified. Especially, it remains unclear whether CALR may act as a biomarker for OSCC patients or not. Thus, we investigated the expression of CALR in 111 OSCC tissue samples using immunohistochemistry to evaluate whether CALR can be a useful biomarker for OSCC in the present study.

We found that among 111 patients with OSCC, CALR expression in tumor cells was low in 67 patients $(60.4 \%)$ and high in 44 patients (39.6\%). In addition, CALR positivity in tumor cell was significantly associated with T classification, $\mathrm{N}$ classification, higher stage and fatal outcome (Table II). Briefly, CALR may be a useful prognostic factor for patients with OSCC according to above our findings. It has been reported that soluble CALR level in sera could be a useful biomarker for detecting lung cancer, and its level in urine might be important for the prediction and diagnosis of bladder cancer $(12,13)$. In addition, overexpression of CALR was correlated with postoperative tumor metastasis in patients with breast cancer (14). Moreover, it was reported that overexpression of CALR might be associated with increased proliferation, migration and angiogenesis of gastric cancer cells (15). Furthermore, elevated expression of CALR was observed in tumor tissues of various cancers including hepatocellular carcinoma (16), colon adenocarcinoma (17) and urinary cancer (13). CALR may be closely associated to tumor malignancy in various cancers.

However, it was also reported that CALR might inhibit growth and/or metastasis of prostate cancer cells in vitro and in vivo (11). Also, cell surface CALR has been considered as an 'eat-me' signal, and cell surface CALR might promote phagocytic uptake of cancer cells by immune systems $(18,19)$. Therefore, the influence of CALR on tumor malignancy may depend on localization of tumor cells as well as cell types and clinical stages. Although there are almost no report on CALR expression affecting OSCC carcinogenesis and patients' outcome, we believe CALR can be a useful biomarker for OSCC.
Our findings showed that high expression of CALR in tumor cell was associated with poor survival of patients (Fig. 2), as well as poor prognosis. Thus, high expression of CALR may promote tumor progression in OSCC. These results suggest that OSCC patients with low expression of CARL may benefit from standard therapy based on surgical resection. Therefore, investigation of CALR expression in biopsy material may lead to select effective treatments for patients with OSCC. In addition, we took the tissue samples from the center of the tumor mass. Sometimes we took a number of samples from different parts of the tumor or different tumors (if they seem heterogeneous) from the same patient if needed, and analyzed them. Therefore, we think our biopsy tissue samples reflected almost all part of the tumors for a particular patient.

This study showed the importance of CALR as a prognostic marker in OSCC. Our data on the correlation of CALR expression levels and $\mathrm{T}$ classification, $\mathrm{N}$ classification, higher stage and fatal outcome were similar to most of the previous reports. In order to clarify the detailed mechanism of action of CALR in OSCC, further investigations are necessary.

This study was funded partly by a Grant-in-Aid (Grant number 16K20581) from the Japanese Ministry of Education, Science and Culture.

All procedures performed in studies involving human participants were in accordance with the ethical standards of the Institutional review board (IRB) of Yamaguchi University Hospital (Ref. H28-057).

For retrospective studies, formal consent from patients is not required. Therefore, informed consent was waived by the IRB.

\section{Acknowledgements}

We thank Dr Dan Cui (Department of Pathology, Yamaguchi University Graduate School of Medicine) for her valuable suggestions and technical support in immunohistochemical analysis. 


\section{References}

1. Cancer Information Service: Cancer Statistics in Japan-2014 http://ganjoho.jp/reg_stat/statistics/brochure/backnumber/2014_ jp.html. Accessed March 14, 2016.

2. National Comprehensive Cancer Network (NCCN): NCCN Clinical Practice Guidelines in Oncology: Head and Neck Cancers, Ver. 1, 2012.http://www.nccn.org/clinical.asp. Accessed March 14, 2016.

3. Inagi K, Takahashi H, Okamoto M, Nakayama M, Makoshi T and Nagai $\mathrm{H}$ : Treatment effects in patients with squamous cell carcinoma of the oral cavity. Acta Otolaryngol Suppl 547: 25-29, 2002.

4. Okada F, Hosokawa M, Hamada JI, Hasegawa J, Kato M, Mizutani M, Ren J, Takeichi N and Kobayashi H: Malignant progression of a mouse fibrosarcoma by host cells reactive to a foreign body (gelatin sponge). Br J Cancer 66: 635-639, 1992.

5. Ishikawa M, Hosokawa M, Oh-hara N, Niho Y and Kobayashi H: Marked granulocytosis in C57BL/6 mice bearing a transplanted BMT-11 fibrosarcoma. J Natl Cancer Inst 78: 567-571, 1987.

6. Kuramitsu Y, Hayashi E, Okada F, Tanaka T,Zhang X, Ueyama Y and Nakamura K: Proteomic analysis for nuclear proteins related to tumour malignant progression: A comparative proteomic study between malignant progressive cells and regressive cells. Anticancer Res 30: 2093-2099, 2010.

7. Kuramitsu Y, Hayashi E, Okada F, Zhang X, Ueyama Y and Nakamura K: Two-dimensional gel electrophoresis using immobilized $\mathrm{pH}$ gradient strips and flamingo ${ }^{\mathrm{TM}}$ fluorescent gel stain identified non-nuclear proteins possibly relates to tumor malignant progression. Anticancer Res 31: 1259-1263, 2011.

8. Hayashi E, Kuramitsu Y, Okada F, Fujimoto M, Zhang X, Kobayashi M, Iizuka N, Ueyama Y and Nakamura K: Proteomic profiling for cancer progression: Differential display analysis for the expression of intracellular proteins between regressive and progressive cancer cell lines. Proteomics 5: 1024-1032, 2005.

9. Sheng W, Chen C, Dong M, Zhou J, Liu Q, Dong Q and Li F: Overexpression of calreticulin contributes to the development and progression of pancreatic cancer. J Cell Physiol 229: 887-897, 2014.
10. Michalak M, Groenendyk J, Szabo E, Gold LI and Opas M: Calreticulin, a multi-process calcium-buffering chaperone of the endoplasmic reticulum. Biochem J 417: 651-666, 2009.

11. Alur M, Nguyen MM, Eggener SE, Jiang F, Dadras SS, Stern J, Kimm S, Roehl K, Kozlowski J, Pins M, et al: Suppressive roles of calreticulin in prostate cancer growth and metastasis. Am J Pathol 175: 882-890, 2009.

12. Liu R, Gong J, Chen J, Li Q, Song C, Zhang J, Li Y, Liu Z, Dong Y, Chen L and Jin B: Calreticulin as a potential diagnostic biomarker for lung cancer. Cancer Immunol Immunother 61: 855-864, 2012.

13. Kageyama S, Isono T, Iwaki H, Wakabayashi Y, Okada Y, Kontani K, Yoshimura K, Terai A, Arai Y and Yoshiki T: Identification by proteomic analysis of calreticulin as a marker for bladder cancer and evaluation of the diagnostic accuracy of its detection in urine. Clin Chem 50: 857-866, 2004.

14. Erić A, Juranić Z, Milovanović Z, Marković I, Inić M, Stanojević-Bakić N and Vojinović-Golubović V: Effects of humoral immunity and calreticulin overexpression on postoperative course in breast cancer. Pathol Oncol Res 15: 89-90, 2009.

15. Chen CN, Chang CC, Su TE, Hsu WM, Jeng YM, Ho MC, Hsieh FJ, Lee PH, Kuo ML, Lee H and Chang KJ: Identification of calreticulin as a prognosis marker and angiogenic regulator in human gastric cancer. Ann Surg Oncol 16: 524-533, 2009.

16. Yoon GS, Lee H, Jung Y, Yu E, Moon HB, Song K and Lee I: Nuclear matrix of calreticulin in hepatocellular carcinoma. Cancer Res 60: 1117-1120, 2000.

17. Brünagel $G$, Shah U, Schoen RE and Getzenberg RH: Identification of calreticulin as a nuclear matrix protein associated with human colon cancer. J Cell Biochem 89: 238-243, 2003.

18. Obeid M, Tesniere A, Ghiringhelli F, Fimia GM, Apetoh L, Perfettini JL, Castedo M, Mignot G, Panaretakis T, Casares $\mathrm{N}$, et al: Calreticulin exposure dictates the immunogenicity of cancer cell death. Nat Med 13: 54-61, 2007.

19. Chao MP, Jaiswal S, Weissman-Tsukamoto R, Alizadeh AA, Gentles AJ, Volkmer J, Weiskopf K, Willingham SB, Raveh T, Park CY, et al: Calreticulin is the dominant pro-phagocytic signal on multiple human cancers and is counterbalanced by CD47. Sci Transl Med 2: 63ra94, 2010. 